\title{
Free-Space Characterization of the Permeability of Inhomogeneous Magneto-Dielectric Materials
}

\author{
Xavier Faget, Amélie Litman, Member, IEEE, Eva Dieudonné, Stefan Enoch, and Nicolas Malléjac
}

\begin{abstract}
A free-space methodology specifically designed for characterizing inhomogeneous magneto-dielectric materials is presented. The associated modeling is performed with the finiteelement method combined with the Born linearized approximation even if the dielectric constants of the materials are known to be large. Due to the introduction of a metallic element, a decoupling of the magnetic and dielectric effects is encountered. This enables us to recover the magnetic characteristic of the material from measured scattered fields, thanks to the inversion of the linear model. The entire methodology is described herein and assessed thanks to an experimental validation.
\end{abstract}

\section{INTRODUCTION}

$\mathbf{I}$ N ADDITION to being exciting structures worth studying, magnetic materials have many applications. Indeed, they are selected as substrate materials in advanced antennas' design [1], [2] or for the development of electromagnetic interference suppression [3], [4]. Furthermore, there is a renewal of interest in these specific materials due to the recent development of metamaterials [5]-[7]. Let us also mention the promising capabilities of cloaking [8], which involves complicated but adequate spatial distributions of magnetodielectric materials [7].

In the design of these various structures, it is of great importance to accurately know the values of the electromagnetic characteristics of these materials. There currently exist numerous ways to measure the permittivity and the permeability of small and machined samples [9]. For example, one can characterize those samples with resonant cavities [10] when one is interested in accurate measurements at a specific frequency value or one can employ coaxial lines [11] for a broadband measurement.

Nevertheless, these techniques fail to provide the spatial heterogeneities more than likely to appear in large-scale samples, even if the fabrication protocols are carefully controlled. It is thus important, from a reverse engineering perspective, to control the actual spatial variations of the finished materials, since unintended heterogeneities might have considerable effects on

Manuscript received January 30, 2017; revised April 28, 2017; accepted June 4, 2017. The work of X. Faget for the Ph.D. and the project was supported by CEA. (Corresponding author: Xavier Faget.)

X. Faget, E. Dieudonné, and N. Malléjac are with the CEA DAM/Le Ripault, BP 16, F-37260 Monts, France (e-mail: gnompom29@gmail.com).

A. Litman and S. Enoch are with the Aix Marseille University, CNRS, Centrale Marseille, Institut Fresnel, Marseille, France.

Color versions of one or more of the figures in this paper are available online at http://ieeexplore.ieee.org.

Digital Object Identifier 10.1109/TMTT.2017.2722402 the performances of the final components. For example, in the cloaking application, it is a real challenge to verify the spatial properties of the heterogeneous cloak structure by means of a nondestructive method. Nonetheless, it is known that a tiny perturbation of the cloak structure would induce a notable field scattering [12].

This paper is therefore a first step toward the nondestructive assessment of unwanted anomalies in manufactured magnetodielectric structures. In the following, we will therefore focus our attention on detecting and characterizing the spatial variations of the magneto-dielectric parameters, knowing that they should fluctuate around an average value, most probably known in advance.

Since 1989 [13], the transmission/reflection method [14] has been used in free-space for the characterization of homogeneous plates. In general, the model is based on two approximations. First, the plate is considered as an infinite slab or a multilayered slab [15]. Second, the impinging wave is assumed to arrive with a normal incidence with respect to the plate. The edge effects are generally decreased with focusing horns and anechoic absorbers. Although this slab model is efficient for the retrieval of homogeneous properties, it is not appropriate for the reconstruction of spatially varying samples. To the best of our knowledge, there is no specific protocol based on the transmission/reflection method capable of retrieving the spatial inhomogeneities of a magneto-dielectric material from a microwave free-space measurement.

In parallel, the microwave tomography (MWT) has seen major progress in the last decades. The measurements are often performed in free space or in a homogeneous medium, with bistatic or multistatic antenna arrangements, enabling various angles of incidence and reception. The geometry, the locations, and the contrasts of the heterogeneities are the main unknowns of the problem. And even if the samples are sometimes assumed to be infinitely long in one particular direction, the scattering due to the edges is taken into account in the modeling.

MWT has been mainly used so far for the characterization of dielectric objects [16], [17], but the retrieval of the permeability by means of MWT has been barely tackled. Occasionally, it has been treated from purely synthetic data [18]-[21]. Although an imaging procedure of dielectric and magnetic targets from experimental dataset has been reported in [22], only some detection results (localization and geometrical features extraction of the magnetic heterogeneities) have been so far presented. 


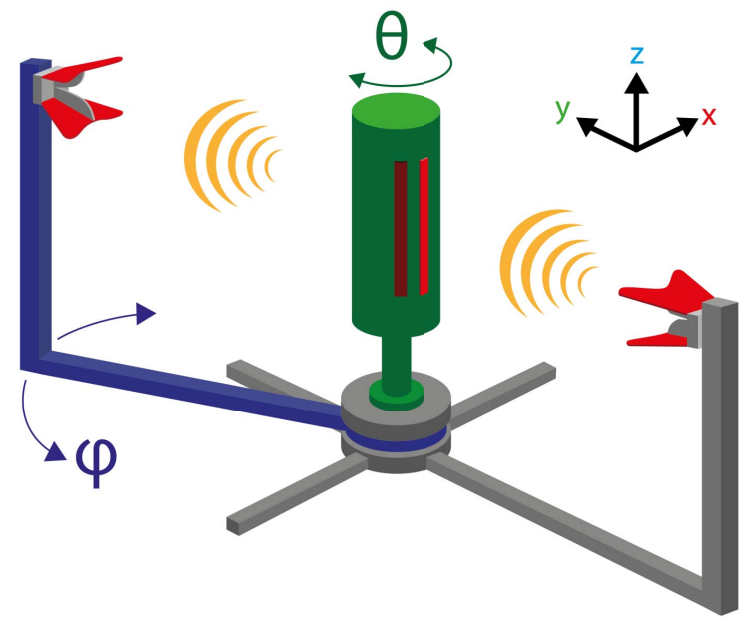

Fig. 1. Sketch of the experimental setup. The target is located at the center of the system. The emitting antenna is mounted on a fixed arm, while the receiving antenna is positioned on a rotating arm. The target can also rotate on itself.

In this paper, we will thus detail a measurement protocol specifically meant for characterizing heterogeneous magnetodielectric structures in free space, where we will focus on the recovery of the spatial variations of the magnetic parameters. To that end, we will adopt an MWT strategy with a bistatic or multistatic measurement configuration. As we are mainly concerned with a reverse engineering application, we will take advantage of the knowledge of the external dimensions and geometries of the samples, which will be included in our finite-element model. In particular, thanks to the reciprocity theorem, this finite-element model enables us to compute the Green's function for any kind of measurement configuration, even if we have restricted ourselves here to a 2-D problem. We will also show that the forward problem can be linearized with the Born approximation, even if the dielectric contrast between the target and the environment is large. Thanks to the clever use of a metallic cylinder, we will also show that we are able to separate the dielectric and magnetic contributions in the target's scattered field. This permits us to recover the permeability characteristics of the inhomogeneous sample from bistatic measurements. Comparisons between simulated and measured fields are provided as well as inversion results.

\section{EXPERIMENTAL SETUP}

\section{A. System Description}

As we can see in Fig. 1, the measurement system consists of a bistatic bench. The system works in a $6 \times 6 \times 3 \mathrm{~m}^{3}$ chamber without microwave absorber walls. A network analyzer PNA-L N5232A is directly connected to both antennas with coaxial high-frequency cables SUCOFLEX 104PEA. The receiving cable crosses the center of the system through a revolving joint, which ensures that the cable does not move during the measurement. The receiving and emitting antennas are model ETS-Lingren 3117 . They are assumed to be linearly polarized and are used only in a single polarization case, parallel to $\vec{z}$. They work between 1 and $18 \mathrm{GHz}$. For this
TABLE I

Electromagnetic Properties of Materials at $3.5 \mathrm{GHz}$

\begin{tabular}{ccc}
\hline & Plate 1 & Plate 2 \\
\hline Permittivity & $6.6( \pm 1.3)-0.23( \pm 0.08) i$ & $8.6( \pm 0.7)-0.3( \pm 0.09) i$ \\
\hline Permeability & $1.5( \pm 0.06)-0.4( \pm 0.08) i$ & $1.7( \pm 0.06)-0.7( \pm 0.07) i$ \\
\hline
\end{tabular}

paper, the two antennas are positioned at a fixed height, on a circle with radius $R=100 \mathrm{~cm}$ from the center of the bench. Measurements are automated with the LabView software, which controls the rotating motors and the network analyzer. A first motor controls the $\varphi$ angle, i.e., the position of the receiving antenna, while the emitting antenna remains at a fixed position. Looking down from the top in Fig. 1, the direction of rotation of $\varphi$ is clockwise. We define $\varphi=0$ when the two antennas are facing each other. A second motor controls the rotation of the target on itself, with a $\theta$ angle. This enables a multistatic configuration.

\section{B. Support}

Contrary to what is currently done in free-space measurements, the target is not simply set on a polystyrene mast. Instead, its support corresponds to a metallic circular cylinder, whose dimensions are $80 \times 12.35 \mathrm{~cm}^{2}$ in height and radius. This support has been carefully chosen as, near the metal, the electric field is weak while the magnetic field is enhanced. This well-known phenomenon is used to exacerbate the contribution of the permeability variations in the scattered field and highly reduces the contribution of the permittivity variations. This support is the key element that enables us to afterward employ a linearized forward model even if the permittivity contrast between the samples and the vacuum is large (see Section V-C). The cylindrical shape of the support has been chosen in order to facilitate the alignment protocol but other geometries, such as, for example, a metallic sheet, could work as well.

\section{Materials}

The samples consist of $60 \times 3 \times 0.08 \mathrm{~cm}^{3}$ magneto-dielectric homogeneous strips produced by a pneumatic spray facility. Two homogeneous plates with different levels of indices are fabricated (Table I) before being machined into strips with a tolerance of less than $0.05 \mathrm{~mm}$. This permits to set aside possible issues associated with the thickness variations. They have been measured using a coaxial-line method [14]. In each of those plates, five samples have been randomly extracted and measured to ascertain the homogeneity of the plates. The measurement uncertainties are smaller than the inhomogeneity found inside a homogeneous strip, and thus we provide only the $95 \%$ confidence interval (CI) associated with the inhomogeneities (Fig. 2).

The permittivity of the materials has been found to be quasiconstant in the considered frequency band. It has been reported in Table I as well as the permeability at $3.5 \mathrm{GHz}$. The given uncertainties correspond to the $95 \%$ CI.

To create an inhomogeneous sample, the strips are vertically glued side to side on the metallic cylinder with a repositionable adhesive spray or with an adhesive layer. In any 

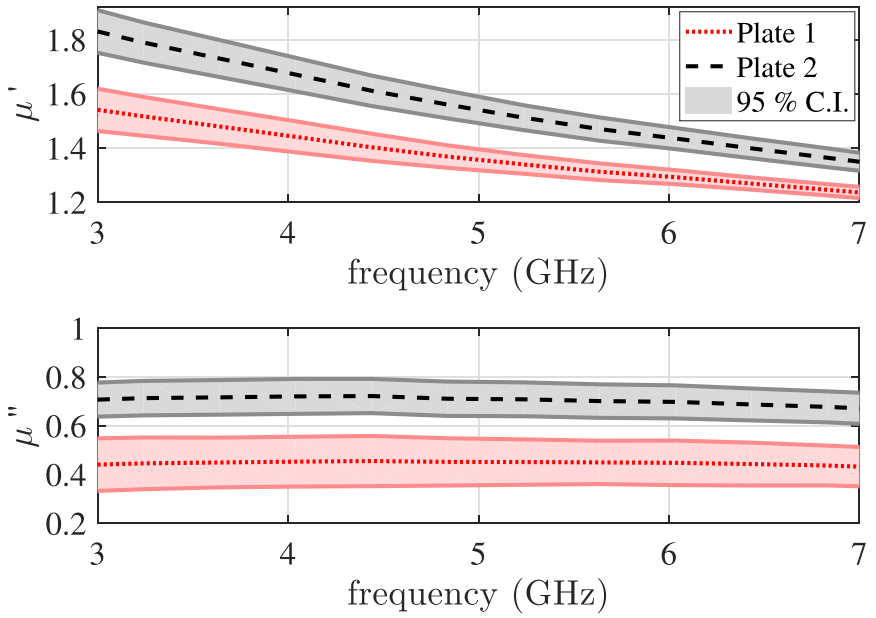

Fig. 2. Variation of the plates' permeability in function of the frequency, with associated CIs, measured with a coaxial-line method.

case, the spacing between a strip and the metallic cylinder due to the adhesive layer is less than $0.06 \mathrm{~mm}$. We have numerically checked that this dielectric gap, the adhesive material being nonmagnetic, has a negligible contribution in the scattering process. As the spray is not powerful enough to oblige the strips to conform to the geometry of the cylinder, they keep their rectangular shape. They will thus be modeled as rectangles in the forward model in order to be as close as possible to the actual geometry. For calibration purposes, we have also machined a $60 \times 3 \times 0.3 \mathrm{~cm}^{3}$ metallic strip.

\section{Data AcQuisition And Processing}

\section{A. Measurement Protocol}

The measurements are realized with two different protocols. In the bistatic case, the metallic support remains fixed while the RX antenna position $\varphi$ varies from $-164^{\circ}$ to $164^{\circ}$, with $N_{r}=329$ equidistant angular steps. In the second protocol, the RX antenna position $\varphi$ varies from $-165^{\circ}$ to $165^{\circ}$, with $N_{r}=56$ equidistant angular steps while the cylinder position $\theta$ varies from $-90^{\circ}$ to $90^{\circ}$ with $N_{e}=9$ equidistant angular steps. The rotation of the cylinder can be equivalently viewed as a displacement of the emitting antenna, creating thus a different incidence angle and therefore a multistatic configuration. The simultaneous rotation of the cylinder and the receiving antenna requires additional time measurements. This is the reason why the number of receiving points has been reduced in the multistatic case. Nevertheless, the information content provided by the multistatic configuration is increased compared with the bistatic case as shown in Section VI-B.

At each emission/reception position, the network analyzer measures $N_{\omega}=1024$ frequency points equally spaced in the frequency band from 2 to $17 \mathrm{GHz}$. Because of the large number of points collected and to reduce the measurement time, we do not perform extra measurements averaging. Due to the polarization of the antennas, only the $z$-component of the electric field is considered. It is denoted by $E_{\text {meas }}$ in the following.

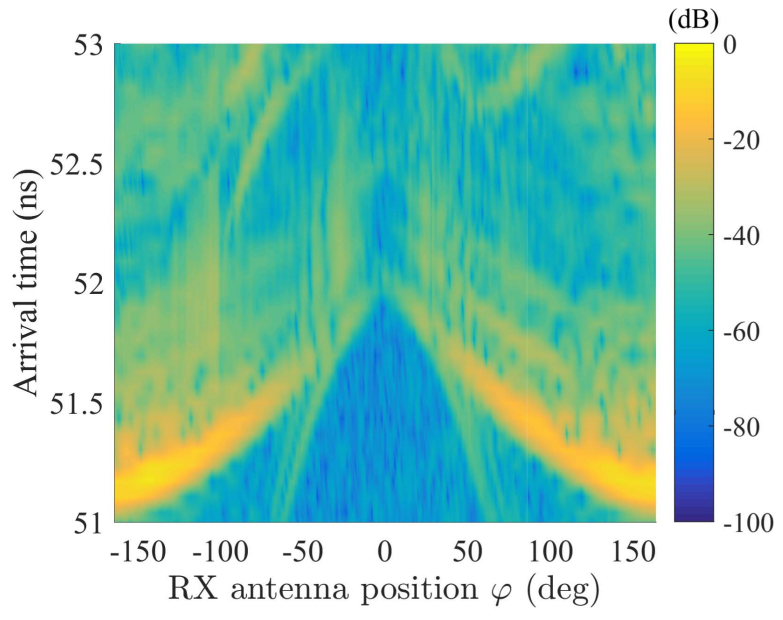

Fig. 3. Radargram of the scattered field measured when nine strips are glued on the metallic cylinder.

\section{B. Temporal Filtering}

For each measurement position, a Hamming window is performed in the spectral domain before applying a Fourier transform to analyze the signal in the temporal domain. The corresponding radargrams are visible in Fig. 3.

Because of the geometry of the experimental system, we have estimated that the signals scattered by the various objects glued on the cylinder have a very short duration (under $2 \mathrm{~ns}$ ) and are located after $51 \mathrm{~ns}$. Everything out of this gate is filtered out with a time gating procedure. This choice is rather drastic as it eliminates additional effects, among which some could be worth exploited (e.g., the creeping waves due to the metallic cylinder and the samples), or worth removed (unexpected wave interactions with the external environment). It must be recalled that the measurements are not performed in an anechoic environment. Thus, there are good chances that extra perturbations will appear in the measured fields (echoes from the surrounding walls, from the network analyzer located inside the room). The underlying idea here is to arrive at a procedure containing a reduced number of tuning parameters and quite robust to external perturbations, even with the possible drawback of losing parts of the information.

After the time gating, an inverse Fourier transform takes back the signal to the frequency domain. A signal normalization is then applied because the gating procedure naturally lowers the signal amplitude (depending on the frequency). Furthermore, the amplitude level of the field scattered by the magneto-dielectric materials, even if it is slightly lower, has the same order of magnitude than the one scattered by the metallic strip, which is used for calibration purposes.

\section{Simulation}

We assume that the objects are isotropic, infinitely long, and spatially invariant along the $z$-axis, even if they are possibly heterogeneous along $x$ and $y$. We further assume that the horn antennas are perfectly linearly polarized and their polarization direction is set along the $z$-axis. These assumptions enable us to keep on with a 2-D formulation. To simplify the notation, we define $E$ as the $z$-component of the electric field $\vec{E}$. All 
the computations are performed in the frequency domain, with a time convention in $\exp (i \omega t)$.

\section{A. Finite-Element Model}

At first, a full-wave model is considered

$$
\left\{\begin{array}{l}
\vec{\nabla} \times \vec{H}-i \omega \varepsilon_{0} \varepsilon_{r} \vec{E}=\vec{J} \\
\vec{\nabla} \times \vec{E}+i \omega \mu_{0} \mu_{r} \vec{H}=\overrightarrow{0}
\end{array}\right.
$$

where $\varepsilon_{0}$ and $\mu_{0}$ are, respectively, the permittivity and the permeability of vacuum. The relative permittivity $\varepsilon_{r}(\vec{r})=$ $\varepsilon_{r}^{\prime}(\vec{r})-i \varepsilon_{r}^{\prime \prime}(\vec{r})$ and permeability $\mu_{r}(\vec{r})=\mu_{r}^{\prime}(\vec{r})-i \mu_{r}^{\prime \prime}(\vec{r})$ vary in space $[\vec{r}=(x, y)]$ according to the materials currently present in the simulation configuration. $\vec{J}$ corresponds to the excitation current, polarized along the $z$-axis.

The equation is solved with a finite-element method, from the COMSOL software. The excitation is generated by an electric current line source positioned at $\vec{r}_{e}=(0,100) \mathrm{cm}$ and is definitely not a plane wave, contrary to what was done in [23]. The geometry is contained in a circle with a radius equal to $110 \mathrm{~cm}$. The numerical mesh is confined with a perfectly matched layer boundary. Metallic components like the cylindrical support or the calibration strip are declared as perfect electric conductors.

This numerical tool permits us to calculate all simulated fields denoted by $E_{\text {fem }}$ and $\vec{H}_{\text {fem }}$ in the following, whose values will vary according to the choice of the spatial distribution of $\varepsilon_{r}$ and $\mu_{r}$ (see Sections II-C and V-A).

\section{B. Linearized Model}

In order to speed up the computation and propose a simple way to perform the inversion step, we investigate the potentiality of a linearized approximation. Even if these expressions are already known [18], we restate them by means of a different approach.

We start by defining two distinct configurations, with or without the magneto-dielectric sample [Fig. 4(a) and (b)]. Whatever the case, the metallic support is always included in the model. Then the scattered fields are defined as

$$
\left\{\begin{array}{l}
\vec{E}^{S}=\vec{E}^{b}-\vec{E}^{a} \\
\vec{H}^{S}=\vec{H}^{b}-\vec{H}^{a} .
\end{array}\right.
$$

Thanks to the volume equivalence theorem [24], the sample when illuminated generates secondary line sources that are defined only on the sample domain $\Omega$. In the configuration depicted in Fig. 4(c), (1) becomes

$$
\left\{\begin{array}{l}
\vec{\nabla} \times \vec{H}^{S}-i \omega \varepsilon_{0} \varepsilon_{r}^{a} \vec{E}^{S}=\vec{J}_{\mathrm{eq}} \\
\vec{\nabla} \times \vec{E}^{S}+i \omega \mu_{0} \mu_{r}^{a} \vec{H}^{S}=-\vec{M}_{\mathrm{eq}}
\end{array}\right.
$$

where $\varepsilon_{r}^{a}(\vec{r})$ and $\mu_{r}^{a}(\vec{r})$ correspond to the relative permittivity and permeability distribution associated with the first configuration, i.e., where only the metallic cylinder that serves as a support for the strips is present [see Fig. 4(c)]. The polarization currents are defined as

$$
\begin{aligned}
\vec{J}_{\mathrm{eq}} & =i \omega \varepsilon_{0}\left[\varepsilon_{r}^{b}-\varepsilon_{r}^{a}\right] \vec{E}^{b}=i \omega \varepsilon_{0} \chi_{\varepsilon} \vec{E}^{b} \\
\vec{M}_{\mathrm{eq}} & =i \omega \mu_{0}\left[\mu_{r}^{b}-\mu_{r}^{a}\right] \vec{H}^{b}=i \omega \mu_{0} \chi_{\mu} \vec{H}^{b}
\end{aligned}
$$

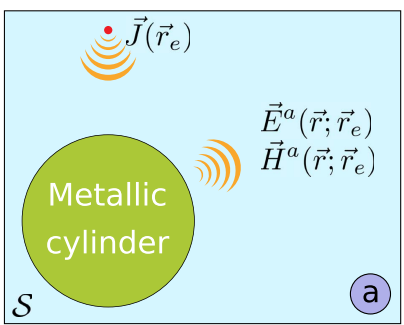

(a)

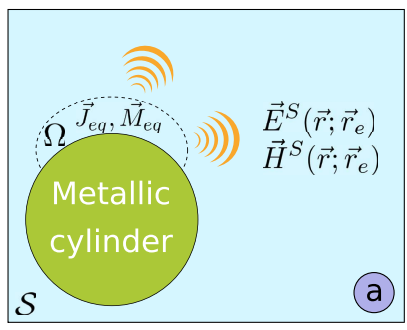

(c)

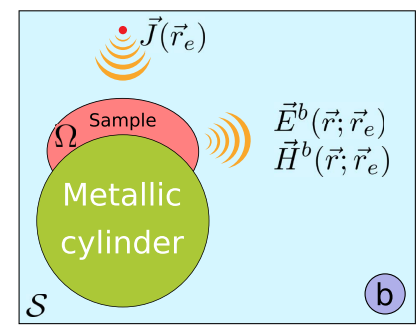

(b)

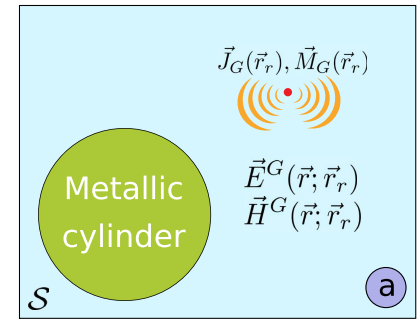

(d)
Fig. 4. Numerical configurations used to establish a linear model. (a) Without the sample. (b) With the sample. (c) Describing the radiation of equivalent line sources. (d) Fictitious configuration in which the receiving antenna radiates. Configurations (a) and (b) are used to compute the scattered field.

where $\chi_{\varepsilon}(\vec{r})$ and $\chi_{\mu}(\vec{r})$ are the well-known contrast functions and are the actual unknowns of the inverse problem, which will be treated later on (see Section VI-B).

In order to establish a linear model, we consider a new computational case [Fig. 4(d)]. It differs only from the first medium [Fig. 4(a)] by the presence of extra line sources $\vec{J}_{G}\left(\vec{r}_{r}\right)$ and $\vec{M}_{G}\left(\vec{r}_{r}\right)$, which are not located on the actual emitting antenna position $\vec{r}_{e}$ but instead on the RX antenna position $\vec{r}_{r}$. The electromagnetic fields associated with such sources will be denoted in the following by $\vec{E}^{G}$ and $\vec{H}^{G}$ and will act as Green's functions linking the field inside the sample to the field at the observation points. Let us now take advantage of the reciprocity theorem [25] to combine the two cases present in Fig. 4(c) and (d)

$$
\left\langle\vec{E}^{S}, \vec{J}_{G}\right\rangle-\left\langle\vec{H}^{S}, \vec{M}_{G}\right\rangle=\left\langle\vec{E}^{G}, \vec{J}_{\mathrm{eq}}\right\rangle-\left\langle\vec{H}^{G}, \vec{M}_{\mathrm{eq}}\right\rangle
$$

where the inner product is defined as $\langle\vec{A}, \vec{B}\rangle=\int_{\mathcal{S}} \vec{A} \cdot \vec{B} d \vec{r}$. This theorem requires that we are in the presence of the same medium but different sources and it is applicable only for reciprocal media. It means that the media can be inhomogeneous, anisotropic, and lossy, but materials have to be described by symmetrical tensors. In our case, they are inhomogeneous and presumably isotropic.

As we do not have access to the magnetic field measurement, we choose to use only electric sources in the Green simulation case in order to suppress the term in $\vec{H}^{S}$. Furthermore, the electric source is chosen as a current source $\vec{J}^{G}=\delta\left(\vec{r}-\vec{r}_{r}\right) \vec{z}$ in order to have a correct correlation between the numerical and the experimental polarization. Replacing all the current sources by their respective expressions in (5), we obtain

$$
\begin{aligned}
E^{S}\left(\vec{r}_{r} ; \vec{r}_{e}\right)= & i \omega \varepsilon_{0} \int_{\mathcal{S}} \chi_{\varepsilon}(\vec{r}) E^{b}\left(\vec{r} ; \vec{r}_{e}\right) E^{G}\left(\vec{r} ; \vec{r}_{r}\right) d \vec{r} \\
& -i \omega \mu_{0} \int_{\mathcal{S}} \chi_{\mu}(\vec{r}) \vec{H}^{b}\left(\vec{r} ; \vec{r}_{e}\right) \cdot \vec{H}^{G}\left(\vec{r} ; \vec{r}_{r}\right) d \vec{r}
\end{aligned}
$$


Unfortunately, in the previous equation, one needs the full knowledge of $\vec{E}^{b}$ and $\vec{H}^{b}$ in order to compute the scattered field. These fields are in turn nonlinearly linked to the permittivity and permeability contrasts $\chi_{\varepsilon}$ and $\chi_{\mu}$. To simplify the computations, the linear Born approximation is employed, even if this approximation is known to be invalid for high contrast or large objects compared with the wavelength [26], [27]. To ensure that the Born approximation remains valid even when the samples have dimensions that are large with respect to the wavelength, one must select the electromagnetic values in the reference scenario not too far from the unknown permittivities and permeabilities of the samples. Fortunately, in the framework of reverse engineering, the average values are known in advance and can be easily incorporated in the reference case. Here, as the samples are thin, we have only considered as reference values the ones corresponding to vacuum.

Because the contrast is null outside the sample domain $\Omega=\left\{\vec{r} \in \mathcal{S}, \varepsilon_{r}^{b}(\vec{r}) \neq \varepsilon_{r}^{a}(\vec{r})\right\}$, the integration is only done on this restricted domain $\Omega$. Finally, we obtain

$$
\begin{aligned}
E_{\operatorname{lin}}^{S}\left(\vec{r}_{r} ; \vec{r}_{e}\right)= & i \omega \varepsilon_{0} \int_{\Omega} \chi_{\varepsilon}(\vec{r}) E^{a}\left(\vec{r} ; \vec{r}_{e}\right) E^{G}\left(\vec{r} ; \vec{r}_{r}\right) d \vec{r} \\
& -i \omega \mu_{0} \int_{\Omega} \chi_{\mu}(\vec{r}) \vec{H}^{a}\left(\vec{r} ; \vec{r}_{e}\right) \cdot \vec{H}^{G}\left(\vec{r} ; \vec{r}_{r}\right) d \vec{r} .
\end{aligned}
$$

This formulation provides a good overview of how physics works. At any point in space, the generated scattered field is a function of three factors : the contrast, the incident field, and the Green function, which depends on the receiver position. If the electric field is really weak in the sample domain, only the permeability will contribute to the scattered field. This is an important point and the main reason of the presence of the metallic cylindrical support as shown in Section V-C.

\section{Numerical Implementation}

In order to evaluate $E_{\operatorname{lin}}^{S}\left(\vec{r}_{r} ; \vec{r}_{e}\right)$, we extract from the finiteelement computation the values of $E_{\mathrm{fem}}^{a}\left(\vec{r} ; \vec{r}_{e}\right), \vec{H}_{\mathrm{fem}}^{a}\left(\vec{r} ; \vec{r}_{e}\right)$, $E_{\mathrm{fem}}^{G}\left(\vec{r} ; \vec{r}_{r}\right)$, and $\vec{H}_{\mathrm{fem}}^{G}\left(\vec{r} ; \vec{r}_{r}\right)$ at each point of the domain $\Omega_{\text {fem }} \supset \Omega$. This domain $\Omega_{\text {fem }}$ has been selected to be slightly larger than the actual support of the strips $\Omega$. It corresponds to a circular ring whose center coincides with the one of the metallic support. Its inner radius is the radius of the metallic support and its width is $4.5 \mathrm{~mm}$. The extraction has been done for $N_{\phi}=4001$ angular steps covering all the ring. At each angular step, $N_{\rho}=46$ radius steps are extracted.

Nevertheless, to further simplify the computational procedure, additional steps are performed before actually computing the integral described in (7). First, the extracted points that are not in the sample domain $\Omega$ are eliminated from the integration. This is the step where the a priori knowledge on the samples external geometries comes into play. Second, each strip is assumed to be radially homogeneous, as the strips are very thin with respect to the wavelength. As the inhomogeneities are depending only on the angle, all cells whose radii share the same angle are combined. As the strips are rigid, they do not fully conform to the metallic support's shape. The extra gap is nevertheless neglected as numerical
TABLE II

Samples Definition (P1: Plate 1, P2: Plate 2, V: VACUUm, AND M: Metallic)

\begin{tabular}{lccccccccc}
\hline Strip number & 1 & 2 & 3 & 4 & 5 & 6 & 7 & 8 & 9 \\
\hline Nine strips & P2 & P1 & P1 & P2 & P1 & P2 & P2 & P1 & P1 \\
Five strips & V & V & P1 & P2 & P1 & P2 & P2 & V & V \\
Two strips & V & V & P2 & V & P1 & V & V & V & V \\
Metallic strip & V & V & V & M & V & V & V & V & V \\
\hline
\end{tabular}

simulations have shown that this additional gap has limited effect on the scattered field. The strips are thus modeled as a thin layer conformal to the cylindrical shape. We then define as many contrasts as requested strips. We set $\Omega=\cup_{d}^{N_{d}} \Omega_{d}$ where $\Omega_{d}$ corresponds to the angular domain related to the $d$ th strip. $N_{d}$ thus corresponds to the number of considered strips where $\chi_{\varepsilon}$ and $\chi_{\mu}$ are known to be nonnull. There is no restriction on the values selected for $N_{d}$, and in particular, there is no reason for it to be equal to the exact number of strips actually present in the sample. The corresponding summation can then be written in matrix format $\mathbf{E}_{\text {lin }}^{S}=\mathbf{G}_{\varepsilon \mu} \boldsymbol{\chi}_{\varepsilon \mu}$

$$
\left[\begin{array}{c}
E_{\operatorname{lin}}^{S}\left(\vec{r}_{1} ; \vec{r}_{1}\right) \\
\vdots \\
E_{\operatorname{lin}}^{S}\left(\vec{r}_{N_{r}} ; \vec{r}_{N_{e}}\right)
\end{array}\right]=\left[\begin{array}{ll}
G_{\varepsilon} & G_{\mu}
\end{array}\right]\left[\begin{array}{c}
\chi_{\varepsilon}^{1} \\
\vdots \\
\chi_{\varepsilon}^{N_{d}} \\
\chi_{\mu}^{1} \\
\vdots \\
\chi_{\mu}^{N_{d}}
\end{array}\right]
$$

with

$$
\begin{aligned}
G_{\varepsilon}^{r d} & =i \omega \varepsilon_{0} \sum_{\vec{r}_{\rho \phi} \in \Omega_{d}} E_{\mathrm{fem}}^{a}\left(\vec{r}_{\rho \phi} ; \vec{r}_{e}\right) E_{\mathrm{fem}}^{G}\left(\vec{r}_{\rho \phi} ; \vec{r}_{r}\right) \Delta_{\rho \phi} \\
G_{\mu}^{r d} & =-i \omega \mu_{0} \sum_{\vec{r}_{\rho \phi} \in \Omega_{d}} \vec{H}_{\mathrm{fem}}^{a}\left(\vec{r}_{\rho \phi} ; \vec{r}_{e}\right) \cdot \vec{H}_{\mathrm{fem}}^{G}\left(\vec{r}_{\rho \phi} ; \vec{r}_{r}\right) \Delta_{\rho \phi}
\end{aligned}
$$

where $\chi_{\varepsilon}^{d}$ and $\chi_{\mu}^{d}$ correspond to the permittivity and permeability contrast in the strip $\Omega_{d} . \Delta_{\rho \phi}$ corresponds to the area of each numerical cell. The integration has thus been performed assuming that the fields and the contrasts are constant in each small radial cell.

\section{Comparison Measurement/Simulation}

\section{A. Samples' Definition}

Several samples have been generated by creating various magneto-dielectric strips' combinations on the surface of the metallic cylinder (see Fig. 5 and Table II). The strips have been mixed in order to create an inhomogeneous repartition.

To minimize possible positioning errors due to multiple manipulations, we perform a predefined measurement sequence, as indicated in Fig. 5.

1) Nine strips are placed side by side on the lateral surface of the cylinder.

2) The four external strips are removed from the cylinder.

3) All the strips are removed.

4) Two strips are glued while not touching each other.

5) The magneto-dielectric strips are removed and replaced by a single metallic strip. 


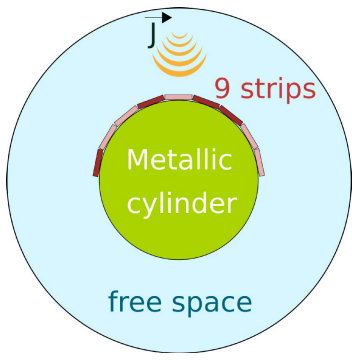

(a)

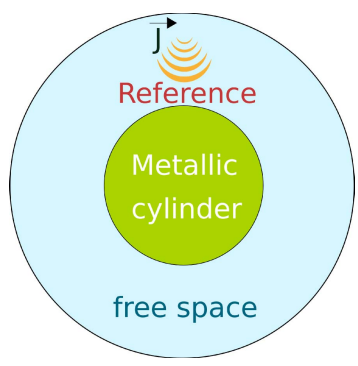

(c)

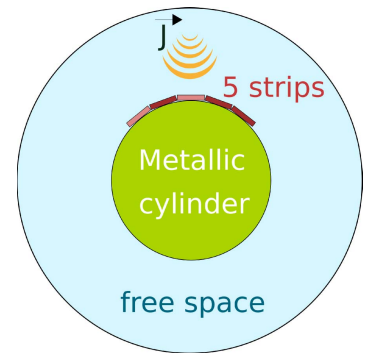

(b)

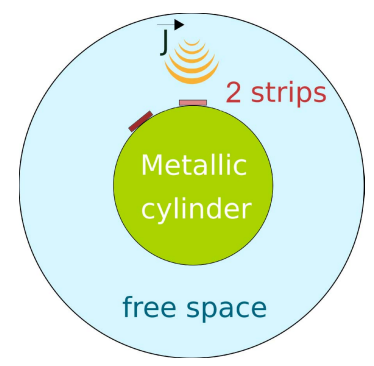

(d)

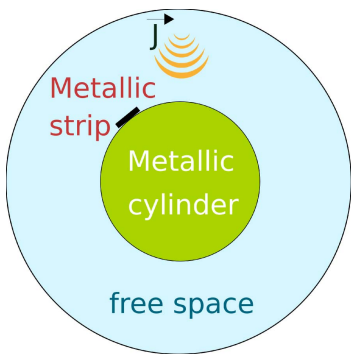

(e)

Fig. 5. Sketch of the various strips' combinations that have been measured and simulated. (a) Nine-strip case. (b) Five-strip case. (c) Empty case. (d) Two-strip case. (e) Metallic strip case.

Of course, every time a new sample is set, its associated electric field is acquired. The last measured field serves during the calibration procedure. The scattered fields are obtained thanks to a complex subtraction between each measured field and the field acquired when there is no strip on the metallic cylinder [Fig. 5(c)]. This empty configuration also corresponds to the simulated one depicted in Fig. 4(a). In the following, only the measured scattered fields $E_{\text {meas }}^{S}$ will be considered.

\section{B. Calibration}

We use the field scattered by the metallic strip to calculate a calibration coefficient, which will enable us to match the simulations to the measurements. We choose to calibrate with a single complex coefficient at each frequency [28] defined as

$$
C(\omega)=\frac{1}{N_{r}} \sum_{r=1}^{N_{r}} \frac{E_{\text {meas }}^{S}\left(\vec{r}_{r} ; \omega\right) \overline{E_{\text {fem }}^{S}\left(\vec{r}_{r} ; \omega\right)}}{\left|E_{\text {meas }}^{S}\left(\vec{r}_{r} ; \omega\right)\right|^{2}}
$$

where $\bar{E}$ stands for the complex conjugate of $E$. This calibration coefficient is applied to any scattered field. In addition to calibrating the field measurements at the simulation level, this coefficient compensates for the 3-D effects that are missing in the simulation but that are nevertheless present in the measurements. It is critically important for the calibration that
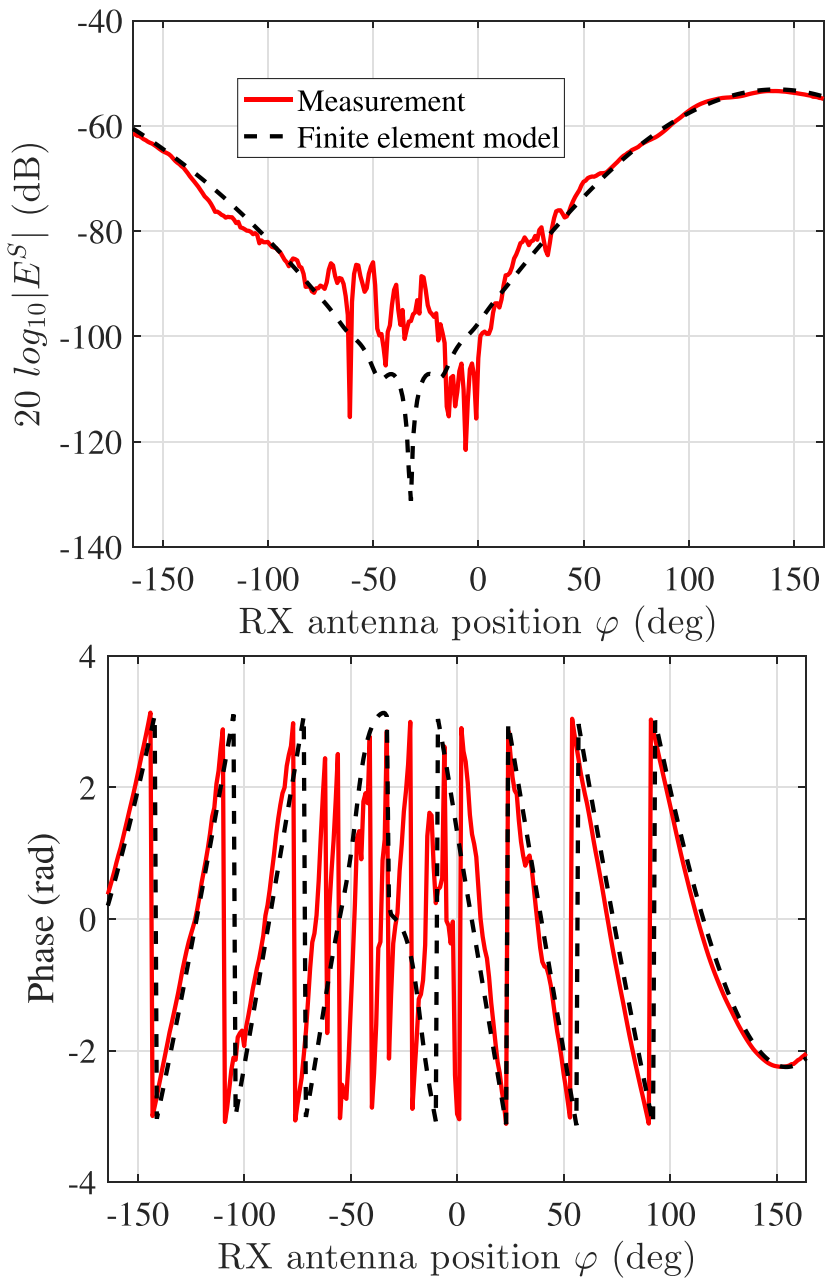

Fig. 6. Amplitude and phase of the scattered field for the metallic strip [Fig. 5(e)] at $3.5 \mathrm{GHz}$ in the bistatic configuration. These two fields are used to compute the calibration coefficient. The calibration coefficient has been applied on the finite-element model.

the metallic calibration strips have the same 2-D size as the measured strips. In Fig. 6, we have plotted the amplitude and the phase of the calibration measurement with its simulation.

We can see that the scattered field is maximal in the backscattering region. In the forward region, the signal is weak and errors are amplified by the logarithm scale. It is worth reminding that, in the scattered field considered here, the main contribution of the metallic cylinder has already been removed, as it is already present in the reference field. This is one of the reasons why there is very little signal in the forward direction, which seems counter-intuitive at first glance. As in that particular angular range, the signal is below the noise floor (estimated to be around $-90 \mathrm{~dB}$ ), only the signal that is acquired at large bistatic angles $(-100<\varphi<40)$ will be used for the calibration procedure. The computed calibration coefficient is consistently applied on the finite-element model and the linearized model.

\section{Field Comparison}

In Fig. 7, we have plotted the measured and simulated fields at $5 \mathrm{GHz}$ for the five-strip case. 

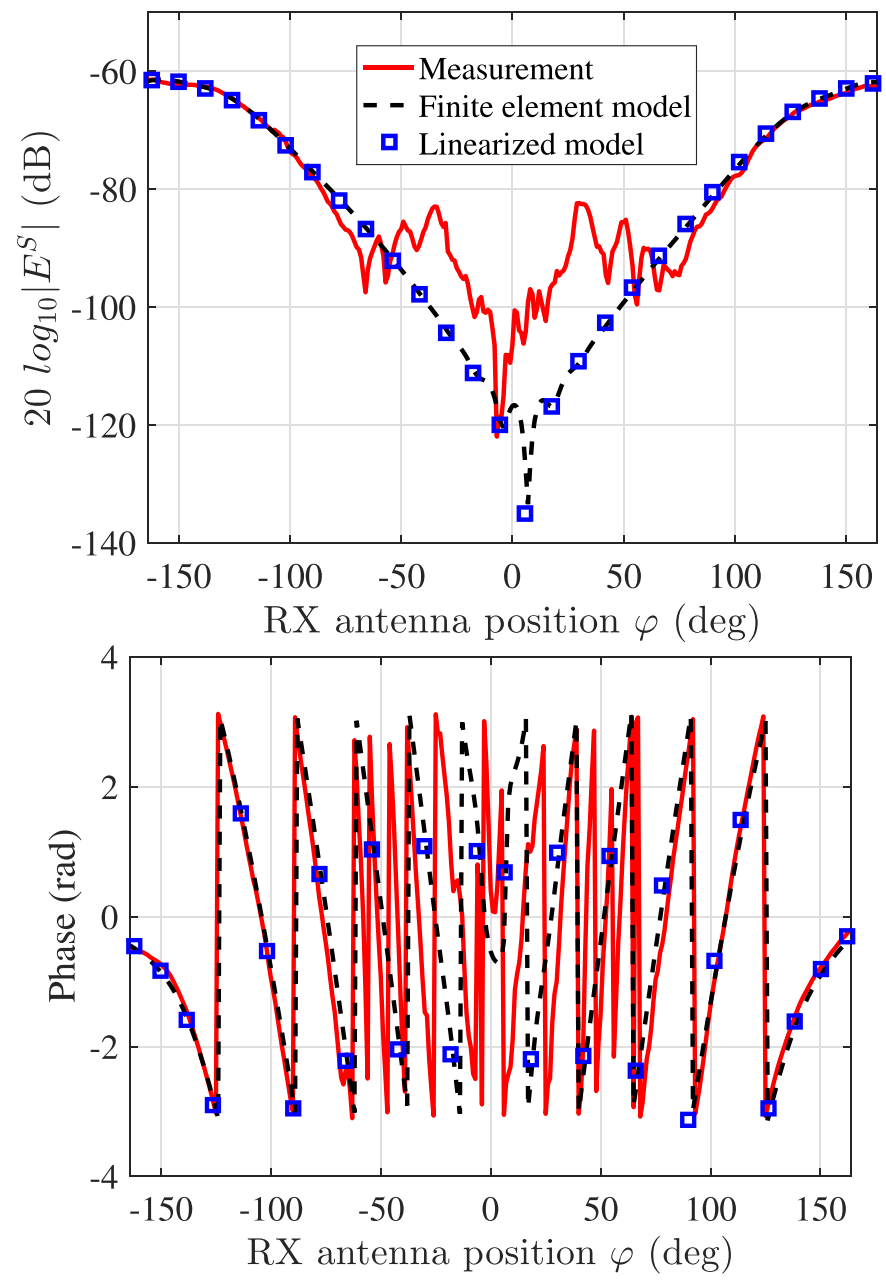

Fig. 7. Amplitude and phase of the measured and simulated scattered fields for the five-strip case [Fig. 5(b)], at $5 \mathrm{GHz}$ in the bistatic configuration.

Despite the fact that there is no anechoic absorber surrounding the measurement setup, these results are showing a good concordance between the simulated fields and the measured ones, both in amplitude and in phase. Of course, the signal level in the forward direction being very weak and thus more sensitive to noise, its associated phase is of limited interest in that particular angular range. Again, only the field acquired at large bistatic angles will be considered in the following. Thankfully, it covers a large enough spread to convey pertinent information related to the samples. This result serves as a good validation of the appropriateness of the measurement and calibration protocol.

It also shows the validity of the linear approximation that has been proposed. Indeed, even if the contrast $\chi_{\varepsilon}$ is large, the Born approximation is valid in this configuration. There are several reasons for that. First, it is due to the small thickness of the samples that have been used. Second, the electric field $\vec{E}^{\text {a }}$ is nearly null in the vicinity of the metallic cylinder (Fig. 8). Thus, in (7), the first integral term is negligible and only the second one, involving the magnetic components, is acting. As on the contrary the permeability contrast $\mu_{r}$ is small, the linear approximation holds.

Finally, Fig. 7 shows us that the experimental noise is bigger than the errors introduced by the linear model. This means that

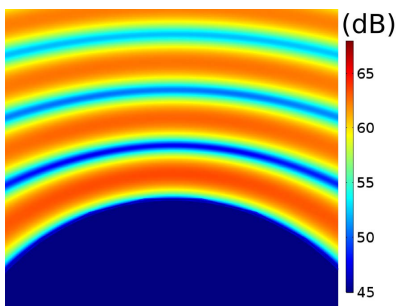

(a)

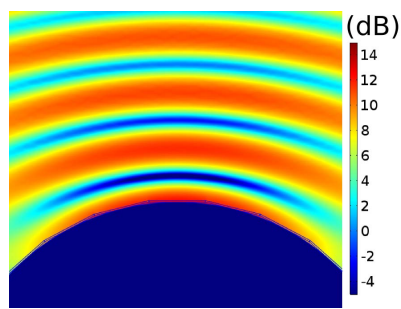

(b)
Fig. 8. (Left) Electric field amplitude $20 \log _{10}\left|E_{\text {fem }}^{a}\right|(\mathrm{dB})$ and (Right) magnetic field amplitude $20 \log _{10}\left|\vec{H}_{\text {fem }}^{a}\right|(\mathrm{dB})$ at the border of the metallic cylinder when nine strips are positioned on the metallic cylinder, at $5 \mathrm{GHz}$. The illumination is coming from the top [Fig. 4(a)].

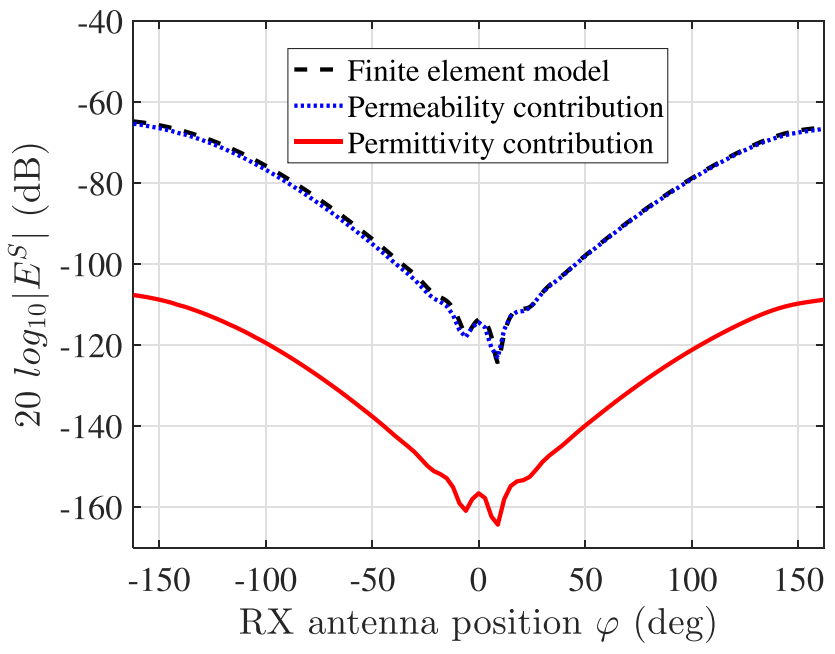

Fig. 9. Comparisons of the amplitude of the scattered field computed with either the magnetic contribution $\mathbf{G}_{\mu} \chi_{\mu}$ or the electric contribution $\mathbf{G}_{\epsilon} \boldsymbol{\chi}_{\epsilon}$ from (8). The scattered field computed with the finite-element model is also plotted. The fields are simulated for the five strips [Fig. 5(b)] at $3.5 \mathrm{GHz}$ in the bistatic configuration.

this linear approach is worth being considered in the present configuration (see Section IV-B).

To have a closer understanding of the influence of the permittivity contribution $\mathbf{G}_{\epsilon} \boldsymbol{\chi}_{\epsilon}$ and the magnetic one $\mathbf{G}_{\mu} \boldsymbol{\chi}_{\mu}$ from (8), we have plotted in Fig. 9 each contribution separately along with the scattered field computed with the full-wave model. As it was expected, the dielectric permittivity contribution is weak and can be neglected. Thus, in the following, we will considered the even simpler linear problem:

$$
\left[\begin{array}{c}
E_{\operatorname{lin}}^{S}\left(\vec{r}_{1} ; \vec{r}_{1}\right) \\
\vdots \\
E_{\operatorname{lin}}^{S}\left(\vec{r}_{N_{r}} ; \vec{r}_{N_{e}}\right)
\end{array}\right]=\left[G_{\mu}\right]\left[\begin{array}{c}
\chi_{\mu}^{1} \\
\vdots \\
\chi_{\mu}
\end{array}\right] .
$$

\section{INVERSION}

\section{A. Linear Inversion Scheme}

The next step is to recover the electromagnetic characteristics of the strips from the measured scattered field. This inverse problem is known to be nonlinear and ill-posed. Thanks to the observations made in Section V-C, we will consider here the linearized version of the scattering problem that is 


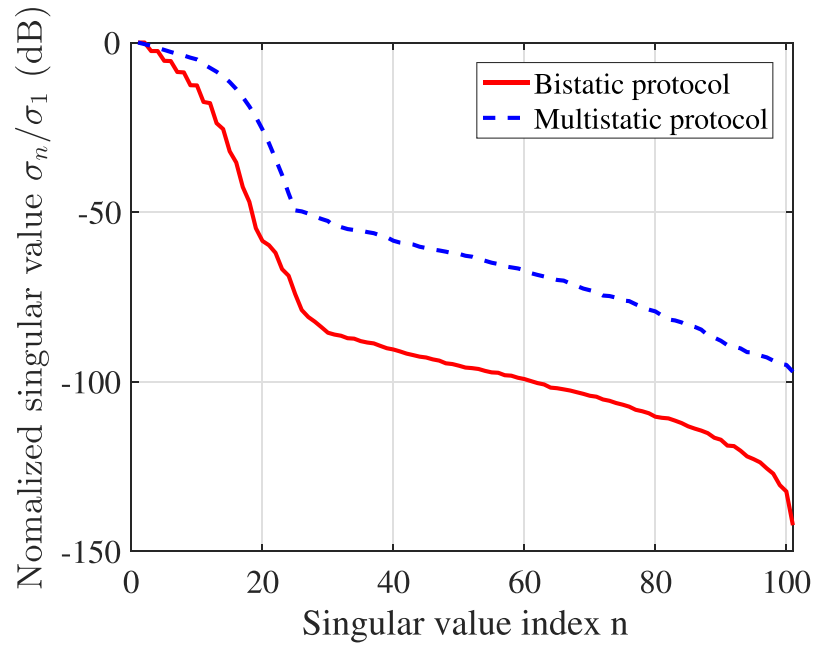

Fig. 10. Spectrum of the matrix $\mathbf{G}_{\mu}$ at $3.5 \mathrm{GHz}$, for the five-strip case. Here $\Omega$ is finely discretized by taking $N_{d}=101$. Values are normalized to the first singular value.

described in (11) and search for the solution of the following minimization problem:

$$
\chi_{\mu}^{*}=\operatorname{argmin}\left\|\mathbf{G}_{\mu} \chi_{\mu}-\mathbf{E}_{\text {meas }}^{S}\right\|^{2}
$$

which corresponds to assuming that the measurement noise is Gaussian [17]. If the error associated with the Born approximation is larger than the experimental noise, advanced nonlinear inversion methods such as the distorted Born iterative method (DBIM) must be considered [29]. In addition, if we have access to the sample average values, the DBIM can be improved using them as initial values as it is an iterative minimization process [30]. In the case of high contrasts, the ill-posedness of the inverse problem can be further reduced by means of the extended-Born approximation and contrastsource formulation as in [31]. The drawback of these nonlinear methods is unfortunately their larger computational burden.

A common way to rapidly evaluate the ill-posedness of this inverse problem is to analyze the spectral behavior of matrix $\mathbf{G}_{\mu}$. We thus perform the singular value decomposition (SVD) of this matrix [32]

$$
\mathbf{G}_{\mu}=\sum_{n} \sigma_{n} \mathbf{u}_{n} \mathbf{v}_{n}^{\dagger}
$$

where $\sigma_{n}$ corresponds to the singular values, $\mathbf{u}_{n}$ the left singular vectors (acting on vectors defined on the receiver locations), and $\mathbf{v}_{n}$ the right singular vectors (acting on vectors defined on the computational domain $\Omega$ ). $\mathbf{v}^{\dagger}$ stands for the transpose conjugate of the vector $\mathbf{v}$.

The singular values are plotted in Fig. 10 and show a significative decay, which is a clear indication of the difficulty related to this inverse problem. In particular, there is a big gap between -20 and $-80 \mathrm{~dB}$ leading to approximatively ten meaningful singular values in the bistatic configuration if the noise level is assumed to be above $-30 \mathrm{~dB}$. Comparatively, in the multistatic configuration, the number of meaningful singular values nearly doubles, which is a clear indication of an increase in terms of information content.
From this spectrum, it is obvious that matrix $\mathbf{G}_{\mu}$ is not of full rank and its pseudoinverse must be considered if one wants to invert (11). Moreover, the rank of $\mathbf{G}_{\mu}$ is not sufficiently large to match the number of unknowns, in particular if the information on the strips geometry is not incorporated in the forward problem formulation. The gathering by strips of the unknowns $\chi_{\mu}$ is thus of great interest to reduce the ill-posedness of the problem.

To compensate for the rank deficiency and the noise present in the measurement, regularization must be applied. Among the well-known robust regularization methods existing for resolving this kind of problem, one can cite the truncated SVD or the Tikhonov regularization [32], [33]. The latter one is favored as the matrix rank is low. It corresponds to solving

$$
\chi_{\mu, \alpha}^{*}=\operatorname{argmin}\left\{\left\|\mathbf{G}_{\mu} \boldsymbol{\chi}_{\mu}-\mathbf{E}_{\text {meas }}^{S}\right\|^{2}+\alpha^{2}\left\|\boldsymbol{\chi}_{\mu}\right\|^{2}\right\}
$$

whose solution is

$$
\chi_{\mu, \alpha}^{*}=\sum_{n=1}^{\min \left(N_{d}, N_{r}\right)} \frac{\sigma_{n}}{\sigma_{n}^{2}+\alpha^{2}} \mathbf{v}_{n} \mathbf{u}_{n}^{\dagger} \mathbf{E}_{\text {meas }}^{S} .
$$

The Tikhonov parameter $\alpha$ controls the weight given to the minimization of the residual norm. If $\alpha$ is too small, the solution is contaminated by the noise and if it is too large, the solution is a poor approximation of the original problem.

Fig. 11 presents the average error committed on the reconstruction in function of the Tikhonov parameter. This error is defined as

$$
\begin{aligned}
& \Delta \mu_{\text {moy }}^{\prime}=\frac{1}{N_{d}} \sum_{d=1}^{N_{d}}\left|\operatorname{Real}\left(\chi_{\mu, \alpha}^{d, *}-\chi_{\mu}^{d, \text { true }}\right)\right|^{2} \\
& \Delta \mu_{\text {moy }}^{\prime \prime}=\frac{1}{N_{d}} \sum_{d=1}^{N_{d}}\left|\operatorname{Im}\left(\chi_{\mu, \alpha}^{d, *}-\chi_{\mu}^{d, \text { true }}\right)\right|^{2}
\end{aligned}
$$

where $\chi_{\mu}^{\text {true }}$ corresponds to the values reported in Table I when the frequency is $3.5 \mathrm{GHz}$. In Fig. 11, the requested tolerance level of 0.25 is also plotted with a dotted line. It shows that there is a large range of values $\alpha$ for which this tolerance is met. It indicates that the proposed approach can robustly provide results that are within the requested bounds. Thanks to [33], we have also considered the L-curve in order to determine a criterion for selecting the Tikhonov parameter when we do not have any knowledge on the sample contrast. In Fig. 11, we can see that the L-curve solution is correct but does not fully correspond to the optimum one. Because the problem is really ill-conditioned, we need to increase the Tikhonov parameter in order to obtain a convergent solution. This is correlated on one side to the error associated with the linearization and on the other side with the experimental noise. Thus, in the following, we have selected a Tikhonov parameter slightly greater than the L-curve solution.

\section{B. Inversion Results}

In Table III, the average error and the max error committed during the inversion are presented for the various configurations with the bistatic protocol at $3.5 \mathrm{GHz}$. 


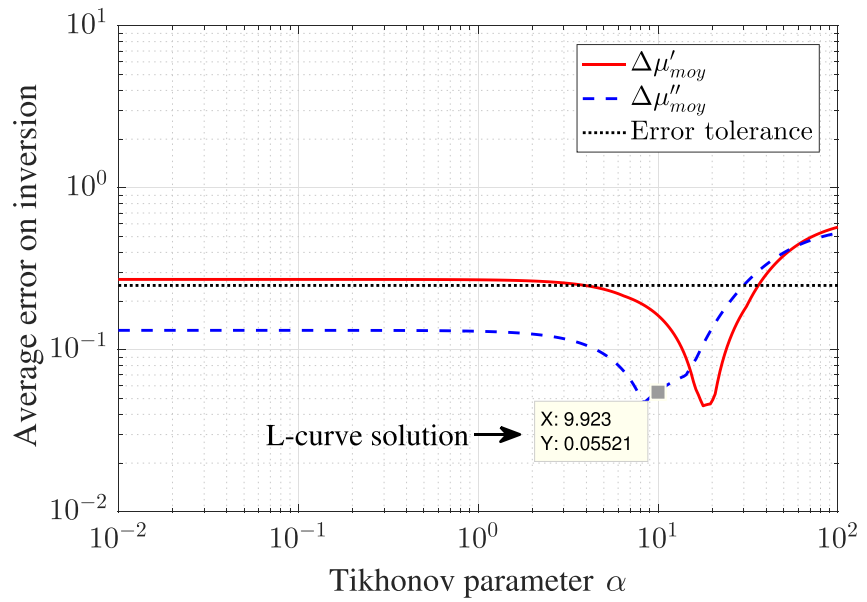

(a)

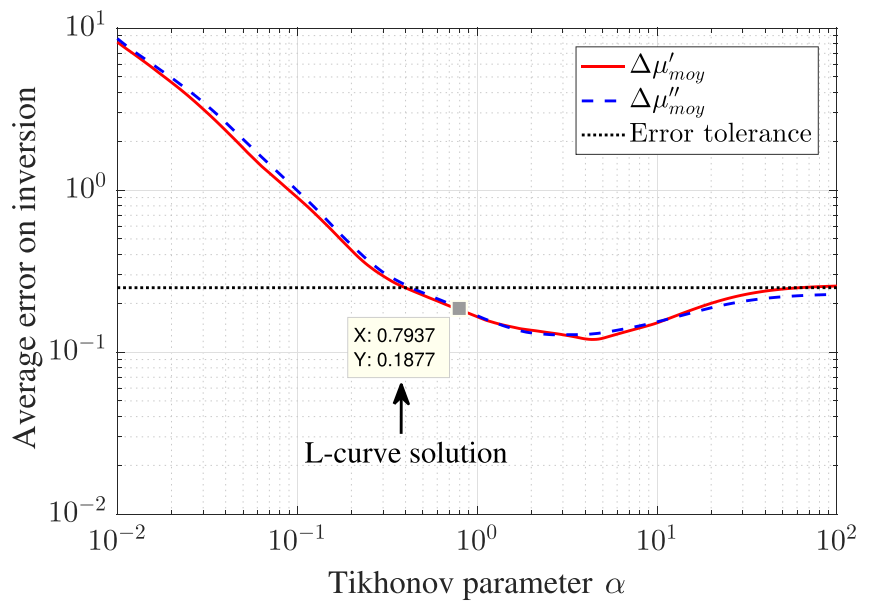

(b)

Fig. 11. Average error committed while reconstructing the five-strip case with the bistatic protocol at $3.5 \mathrm{GHz}$ for various Tikhonov regularization parameters $\alpha$. The number of strips in the inversion is either corresponding to (a) exact number of strips $\left(N_{d}=5\right)$ or (b) very fine discretization $\left(N_{d}=101\right)$. The requested tolerance level is also plotted in dotted line. The L-curve point is also indicated.

TABLE III

ESTIMATION ERror ON THE PERMEABILITy fOR THE VARIOUS SAMPLES IN THE BisTATIC CASE $\left(N_{d}=\right.$ THE EXACT NUMBER OF STRIPS $)$

\begin{tabular}{c|ccc|cc}
\hline Frequency & \multicolumn{3}{|c|}{$3.5 \mathrm{GHz}$} & \multicolumn{2}{c}{$5 \mathrm{GHz}$} \\
\hline Configuration & 2 strips & 5 strips & 9 strips & 5 strips & 9 strips \\
\hline$\Delta \mu_{\text {moy }}^{\prime}$ & 0.011 & 0.087 & 0.065 & 0.075 & 0.120 \\
$\Delta \mu_{\operatorname{moy}}^{\prime \prime}$ & 0.043 & 0.093 & 0.082 & 0.074 & 0.151 \\
\hline$\Delta \mu_{\max }^{\prime}$ & 0.012 & 0.149 & 0.085 & 0.193 & 0.282 \\
$\Delta \mu_{\max }^{\prime \prime}$ & 0.064 & 0.154 & 0.135 & 0.217 & 0.307 \\
\hline
\end{tabular}

In Fig. 12, the inversion results of the five strips case are presented at $3.5 \mathrm{GHz}$ for both protocols. In all these cases, the reconstructed permeability is reasonably located within the requested tolerance. The standard deviations associated with the reconstructed values are not exceedingly accurate, but nevertheless stay within reasonable levels. As we have seen in Fig. 11, the solution depends on the Tikhonov parameter. According to the choice of this parameter, the match will be better for the real part or for the imaginary part of $\mu_{r}$.
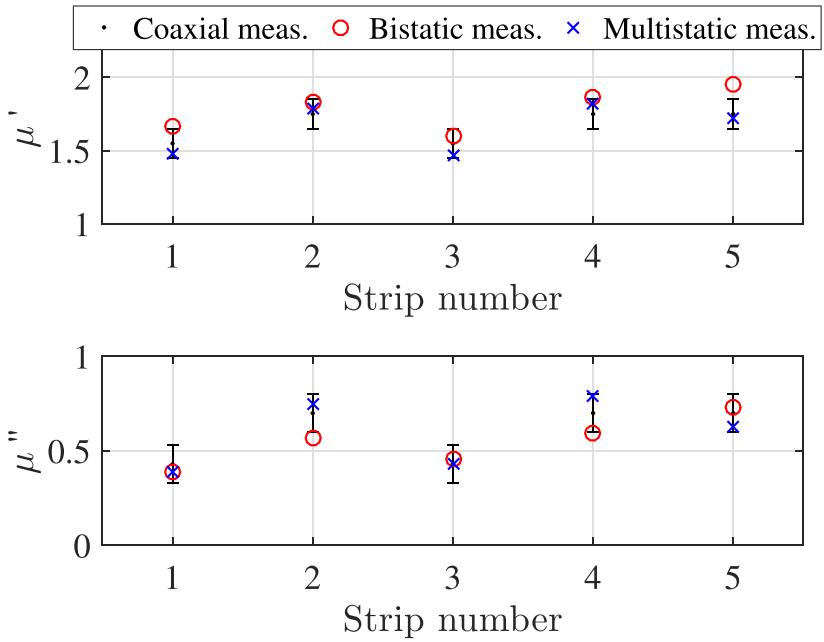

(a)
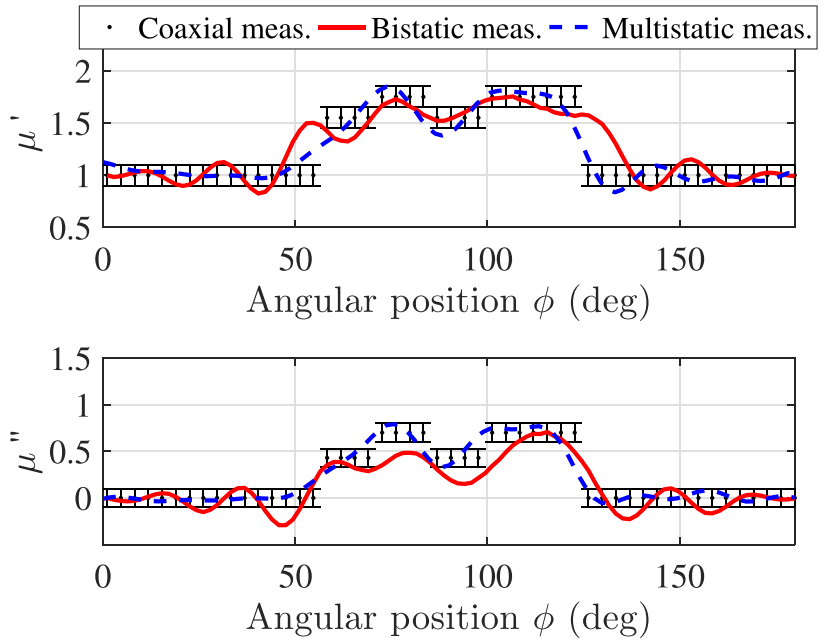

(b)

Fig. 12. Inversion results for the five-strip case [Fig. 5(b)] at $3.5 \mathrm{GHz}$. The number of strips in the inversion is either corresponding to (a) exact number of strips $\left(N_{d}=5\right)$ or (b) very fine discretization $\left(N_{d}=101\right)$.

Even if we consider a fine discretization of $\Omega$, we obtain a good concordance between the reconstructed values and the exact ones. This is worth noting as the expected spatial resolution in the considered configuration is much higher than the discretization step obtained when $N_{d}=101$. Of course, when $N_{d}$ exactly matches with the actual number of strips, the inversion results are improved as the inversion problem becomes less ill-posed. Thus, the proposed methodology enables us to follow the angular spatial variations of the permeability parameter, even without knowing in advance its angular distribution.

When $N_{d}$ corresponds to the actual number of strips $\left(N_{d}=5\right)$, the bistatic results and the multistatic results are somewhat similar. The small differences mainly observed on the imaginary part can be interpreted as measurement bias, in particular due to repositioning errors as the two sets of measurement were not performed at the same time. When the number of unknowns increases $\left(N_{d}=101\right)$, any additional information is beneficial to the inversion process. This is the reason why, as highlighted in Fig. 10, the multistatic approach 

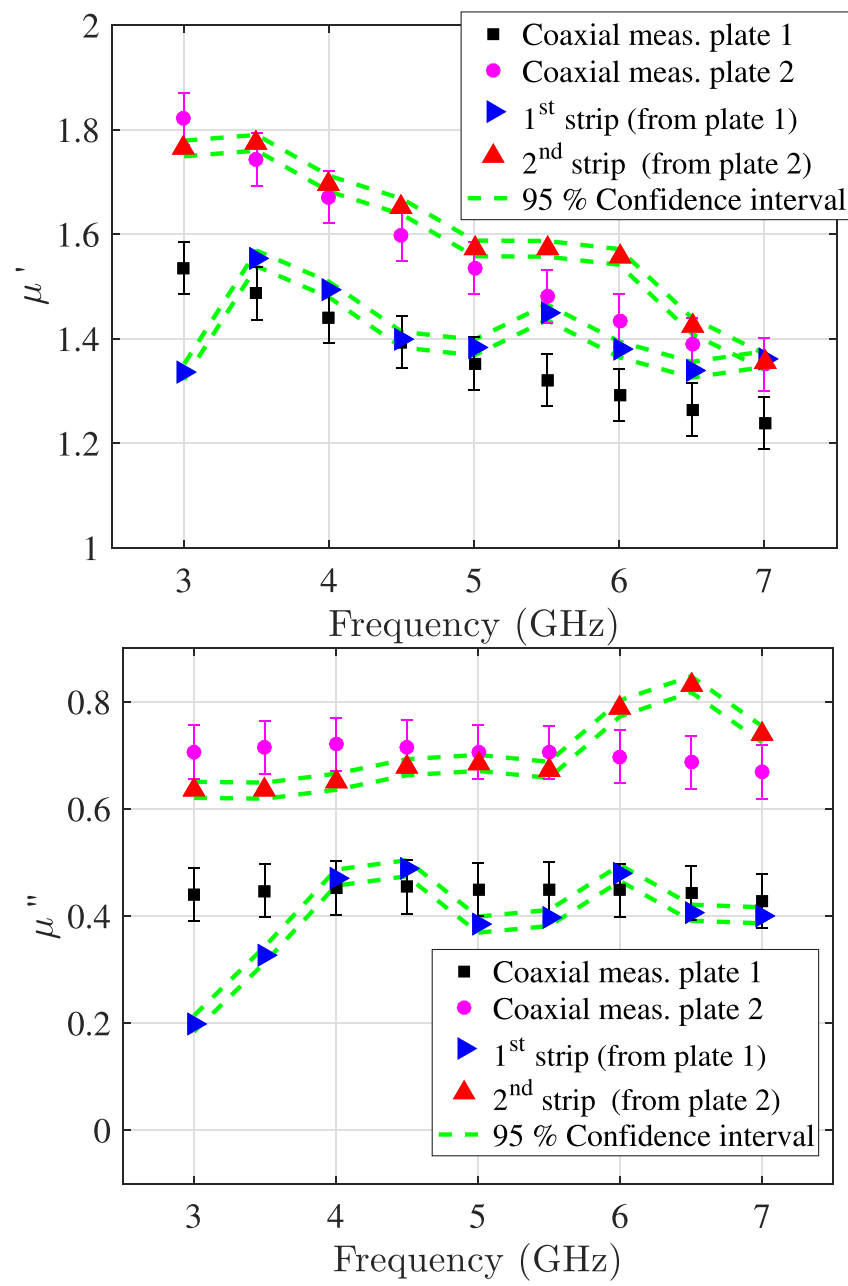

Fig. 13. Inversion results for the five-strip case [Fig. 5(b)] in the bistatic case in function of the frequency. The number of unknowns in the inversion corresponds to the exact number of strips $\left(N_{d}=5\right)$. Only the values of the two first strips are presented.

provides better results than the bistatic one, in particular with less oscillations in homogeneous zones.

We have also performed inversions at several frequencies. The five-strip case has thus been resolved from 3 to $7 \mathrm{GHz}$ with a $0.5 \mathrm{GHz}$ step from bistatic measurements (Fig. 13). For reasons of clarity, only the results of the first two strips are presented. The generalized cross-validation method [33] has been used in order to select automatically the Tikhonov parameter at each frequency. The associated CI is obtained from repeatability measurements. Unfortunately, it does not take into account positioning and calibration errors. The agreement between the coaxial measurements and the inversion results is quite satisfactory on a large frequency range. The discrepancies observed at low or high frequency could be further improved once fully assessing the positioning and calibration errors, following, for example, the methodology employed in [28].

\section{CONCLUSION}

We have presented a new approach for characterizing the permeability of inhomogeneous magneto-dielectric materials in free space from bistatic and multistatic measurements.
To that end, we have positioned magneto-dielectric strips on a metallic circular cylinder in order to decouple the effects linked with the permittivity and the permeability electromagnetic parameters. Moreover, the knowledge of this metallic cylinder has been incorporated inside the modeling and in the definition of the scattered field in order to fully take into account the interaction between the samples and their support. We have experimentally demonstrate that, even with a limited measurement precision due to a basic measurement system deprived of anechoic absorbers and a linearized model, it is possible to recover the permeability characteristics with values that are inside the requested tolerance bounds. In particular, the nonanechoicity aspect is compensated for in part by a rather crude time-gating procedure.

The advantage of this process is the flexibility on the geometry. Indeed, a wide variety of geometries can be considered as long as the samples receive enough illumination. Despite of the relative low spatial resolution linked to the fact that we assume that the parameters are angularly homogeneous by parts, it could permit to retrieve the spatial variations of the permeability within the materials.

\section{ACKNOWLEDGMENT}

The authors would like to thank J. L. Neau and A. L. Adenot-Engelvin for the magneto-dielectric samples that they have prepared. The authors would also like to thank J. H. Le-Gallou and M. Ledieu for their advice.

\section{REFERENCES}

[1] D. M. Pozar and V. Sanchez, "Magnetic tuning of a microstrip antenna on a ferrite substrate," Electron. Lett., vol. 24, no. 12, pp. 729-731, Jun. 1988.

[2] D. Rialet, A. Sharaiha, A.-C. Tarot, and C. Delaveaud, "Estimation of the effective medium for planar microstrip antennas on a dielectric and magnetic truncated substrate," IEEE Antennas Wireless Propag. Lett., vol. 11, pp. 1410-1413, Nov. 2012.

[3] V. G. Harris et al., "Recent advances in processing and applications of microwave ferrites," J. Magn. Magn. Mater, vol. 321, no. 14, pp. 2035-2047, Jul. 2009.

[4] J. Neige, T. Lepetit, A.-L. Adenot-Engelvin, N. Malléjac, A. Thiaville, and N. Vukadinovic, "Microwave permeability of fenimo flakes-polymer composites with and without an applied static magnetic field," IEEE Trans. Magn., vol. 49, no. 3, pp. 1005-1008, Mar. 2013.

[5] J. B. Pendry, A. J. Holden, D. J. Robbins, and W. J. Stewart, "Magnetism from conductors and enhanced nonlinear phenomena," IEEE Trans. Microw. Theory Techn., vol. 47, no. 11, pp. 2075-2084, Nov. 1999.

[6] O. Acher, "Copper vs. Iron: Microwave magnetism in the metamaterial age," J. Magn. Magn. Mater, vol. 321, no. 14, pp. 2093-2101, Jul. 2009.

[7] D. Schurig et al., "Metamaterial electromagnetic cloak at microwave frequencies," Science, vol. 314, no. 5801, pp. 977-980, Oct. 2006.

[8] J. B. Pendry, D. Schurig, and D. R. Smith, "Controlling electromagnetic fields," Science, vol. 312, pp. 1780-1782, Jun. 2006.

[9] L. F. Chen, C. K. Ong, C. P. Neo, V. V. Varadan, and V. K. Varadan, Microwave Electronics: Measurement and Materials Characterization. Hoboken, NJ, USA: Wiley, 2004.

[10] W. E. Courtney, "Analysis and evaluation of a method of measuring the complex permittivity and permeability microwave insulators," IEEE Trans. Microw. Theory Techn., vol. MTT-18, no. 8, pp. 476-485, Aug. 1970.

[11] N.-E. Belhadj-Tahar, A. Fourrier-Lamer, and H. de Chanterac, "Broadband simultaneous measurement of complex permittivity and permeability using a coaxial discontinuity," IEEE Trans. Microw. Theory Techn., vol. 38, no. 1, pp. 1-7, Jan. 1990.

[12] Z. Ruan, M. Yan, C. W. Neff, and M. Qiu, "Ideal cylindrical cloak: Perfect but sensitive to tiny perturbations," Phys. Rev. Lett., vol. 99, no. 11, p. 113903 , Sep. 2007. 
[13] D. Ghodgaonkar, V. Varadan, and V. K. Varadan, "A free-space method for measurement of dielectric constants and loss tangents at microwave frequencies," IEEE Trans. Instrum. Meas., vol. 37, no. 3, pp. 789-793, Jun. 1989.

[14] A. M. Nicolson and G. F. Ross, "Measurement of the intrinsic properties of materials by time-domain techniques," IEEE Trans. Instrum. Meas., vol. IM-19, no. 4, pp. 377-382, Nov. 1970.

[15] D. R. Smith, D. C. Vier, T. Koschny, and C. M. Soukoulis, "Electromagnetic parameter retrieval from inhomogeneous metamaterials," Phys. Rev. E, Stat. Phys. Plasmas Fluids Relat. Interdiscip. Top., vol. 71, pp. 036617-1-036617-11, Mar. 2005.

[16] C. Eyraud, J.-M. Geffrin, A. Litman, and H. Tortel, "Complex permittivity determination from far-field scattering patterns," IEEE Antennas Wireless Propag. Lett., vol. 14, pp. 309-312, Oct. 2014.

[17] C. Eyraud, J.-M. Geffrin, and A. Litman, "3D-aggregate quantitative imaging: Experimental results and polarization effects," IEEE Trans. Antennas Propag., vol. 59, no. 4, pp. 1237-1244, Oct. 2011.

[18] A. Abubakar and P. M. van den Berg, "Iterative forward and inverse algorithms based on domain integral equations for 3-D electric and magnetic objects," J. Comput. Phys., vol. 195, no. 1, pp. 236-262, Mar. 2004.

[19] B. M. Brown and M. Jais, "A variational approach to an electromagnetic inverse problem," Inverse Prob., vol. 27, no. 4, Mar. 2011, Art. no. 045011 .

[20] W. Zhang and Q. H. Liu, "3-D scattering and inverse scattering from objects with simultaneous permittivity and permeability contrasts," IEEE Trans. Geosci. Remote Sens., vol. 53, no. 1, pp. 429-439, Jan. 2015.

[21] M. Gustafsson and S. He, "An optimization approach to 2-D time domain electromagnetic inverse problems," Radio Sci., vol. 35, no. 2 , pp. 525-536, Mar. 2000.

[22] R. Persico, S. Negri, F. Soldovieri, and E. Pettinelli, "Pseudo 3D imaging of dielectric and magnetic anomalies from GPR data," Int. J. Geophys. vol. 2012, p. 512789, Jun. 2012.

[23] P. M. van den Berg, A. Van Broekhoven, and A. Abubakar, "Extended contrast source inversion," Inverse Prob., vol. 15, no. 5, p. 1325, 1999.

[24] C. A. Balanis, Advanced Engineering Electromagnetics. Hoboken, NJ, USA: Wiley, 2012.

[25] E. Dieudonné, N. Malléjac, and S. Enoch, "Scattering by complex inhomogeneous objects: A first-order reciprocity method," Opt. Exp. vol. 22, no. 13, pp. 16558-16570, Jun. 2014.

[26] R. E. Kleinman, G. F. Roach, and P. M. van den Berg, "Convergent born series for large refractive indices," J. Opt. Soc. Amer. A, Opt. Image Sci. vol. 7, no. 5, pp. 890-897, May 1990.

[27] M. Diong, A. Roueff, P. Lasaygues, and A. Litman, "Impact of the born approximation on the estimation error in 2D inverse scattering," Inverse Problems, vol. 32, no. 6, Apr. 2016, Art. no. 065006.

[28] J.-M. Geffrin, C. Eyraud, A. Litman, and P. Sabouroux, "Optimization of a bistatic microwave scattering measurement setup: From high to low scattering targets," Radio Sci., vol. 44, no. 2, p. RS2007, Mar. 2009.

[29] W. C. Chew and Y.-M. Wang, "Reconstruction of 2-D permittivity distribution using the distorted born iterative method," IEEE Trans. Med. Imag., vol. 9, no. 2, pp. 218-225, Jun. 1990

[30] A. Zakaria, A. Baran, and J. LoVetri, "Estimation and use of prior information in fem-csi for biomedical microwave tomography," IEEE Antennas Wireless Propag. Lett., vol. 11, pp. 1606-1609, Jan. 2013

[31] T. Isernia, L. Crocco, and M. D'Urso, "New tools and series for forward and inverse scattering problems in lossy media," IEEE Geosci. Remote Sens. Lett., vol. 1, no. 4, pp. 327-331, Oct. 2004.

[32] M. Bertero and P. Boccacci, Introduction to Inversev Problems in Imaging. Boca Raton, FL, USA: CRC Press, 1998.

[33] P. C. Hansen, "Regularization tools version 4.0 for Matlab 7.3," Numer. Algorthms, vol. 46, no. 2, pp. 189-194, Nov. 2007.

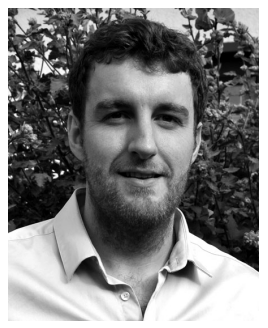

Xavier Faget was born in Caen, France, in 1989. He received the M.S. degree in nonlinear physics from the University of Tours, Tours, France, in 2014. He is currently pursuing the Ph.D. degree in electronic engineering at Aix-Marseille University, Marseille, France, and CEA Le Ripault, Monts, France.

$\mathrm{He}$ is involved with the development of a 2-D free-space measurement system whose purpose is the nondestructive characterization of manufactured magnetodielectric structures. His current research interests include innovative techniques for 3 -D imaging.

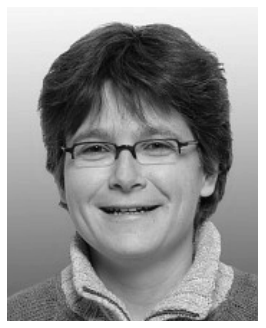

Amélie Litman (M'05) was born in France, in 1972. She received the Ph.D. degree in applied mathematics from the University of Paris XI, Paris, France, in 1997.

From 1997 to 1998, she held a post-doctoral position with the Eindhoven University of Technology, Eindhoven, The Netherlands. From 1998 to 2002, she was with Schlumberger, France, where she was involved in the development of inversion algorithms for oil prospection. In 2002, she joined the Institut Fresnel, Marseille, France, Aix-Marseille University, Marseille, CNRS, and Centrale Marseille, Marseille, as an Assistant Professor. Her current research interests include forward and inverse scattering techniques.

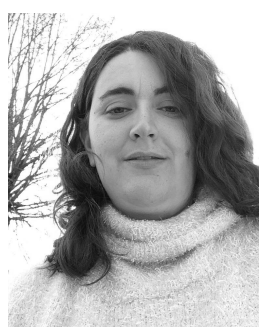

Eva Dieudonné was born in Tours, France, in 1988. She received the M.S. degree in nonlinear physics from the University of Tours, Tours, in 2011, and the $\mathrm{Ph} . \mathrm{D}$. degree in electronics and computer science from Aix-Marseille University, Marseille, France, in 2015 .

In 2015, she joined the GeePs Laboratory, Paris, France, as a Post-Doctoral Researcher, where she is involved with radio-frequency communication between a launcher and the mission control. Her current research interests include new finite element models coupled with the diffraction theory.

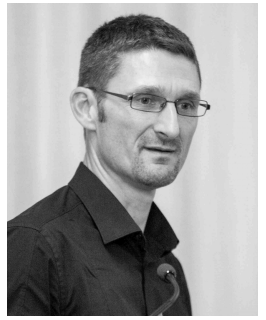

Stefan Enoch received the Ph.D. degree in 1997.

He was an Assistant Professor with Aix-Marseille University, Marseille, France, in 1998. He joined CNRS, Centrale Marseille, Marseille, in 2001, where he is currently a Senior Researcher. He has been the Director of the Institut Fresnel, Marseille, since 2012. He is involved in the theoretical and numerical study of photonic crystals, metamaterials, and plasmonic. He has authored over 120 peerreviewed papers.

Dr. Enoch is a member of the Editorial Board of the Journal of Modern Optics and was an Associate Editor of Optics Express from 2006 to 2012. He was a recipient of the Bronze Medal of the CNRS in 2006 .

Nicolas Malléjac received the engineer's degree from the Ecole Nationale Supérieure d'Informatique et de Mathématiques Appliquées, Saint-Martind'Hères, France, in 1992, and the Ph.D. degree in computer science from the University of Rennes 1, Rennes, France, in 1998.

In 1998, he joined CEA Le Ripault, Monts, France, where he is involved in the modelization and the design of materials and their characterization. 